\title{
PRELIMINARY RISK ASSESSMENT STUDY - NEUROBIOLOGICAL EFFECTS IN EXPERIMENTAL LONG-TIME EXPOSURE TO LOW GSM RADIATION
}

\author{
Soimita Suciu', Dana Dabala ${ }^{2}$, Adrian Florea ${ }^{3}$, \\ Alexandra Sevastre-Berghian', Emanoil Surducan ${ }^{4}$, Vasile Surducan ${ }^{4}$, Camelia Neamtu $^{4}$
}

${ }^{1}$ University of Medicine and Pharmacy Cluj-Napoca-Physiology Department, Cluj-Napoca, Romania

2Railways Medical Clinic Cluj-Napoca-Department of Occupational Medicine, Cluj-Napoca, Romania 3University of Medicine and Pharmacy Cluj-Napoca-Department of Cell and Molecular Biology, Cluj-Napoca, Romania

4National Institute for Research and Development of Isotopic and Molecular Technologies (INCDTIM), Cluj-Napoca, Romania

\begin{abstract}
Purpose: Due to the continuously rising number of mobile phone users of an increasingly younger age, our preliminary study aims to assess the possible neurobiological effects of chronic exposure to microwaves possessing frequencies and power levels similar to GSM signals. For this purpose, rats were irradiated in their daily habitat. Materials and Methods: Twenty male Wistar rats (3 months old) were exposed to GSM 860-89o MHz for 4 hours a day for 36 weeks. This group was compared with sham-exposed rats. The medium exposure value of the microwave field power density was $\approx 60 \mathrm{~mW} / \mathrm{m} 2$ and medium whole body $S A R \approx 0.15 \mathrm{~W} / \mathrm{kg}$. Two types of behavioral tests (open field test and elevated pulse maze) and a transmission electron microscopy on brain samples were performed after 3 and 9 months of exposure, respectively. Results: Exposed rats exhibited decreased locomotor activity and increased emotionality as compared with sham-exposed animals. Transmission electron microscopy examination, performed after 3 and 9 months of exposure, showed neurodegenerative alterations in the hippocampus and the frontal cortex. Severity of the alterations seems to be related to the duration of exposure. Conclusions: These preliminary results suggest that long-term and low-dose cumulative microwave radiation could cause, in rats, ultrastructural changes in neurons, glia and stress behaviour. Further research is needed to investigate the interaction between mobile phone radiations and the central nervous system at the molecular level.
\end{abstract}

Keywords: Behavioral tests, low power GSM, neurodegenerative damage, increased emotionality, transmission electron microscopy

\section{INTRODUCTION}

Biological effects of mobile phone radiation are the subjects of several decades' worth of scientific research and the intense debate regarding whether or not phone radiation is harmful to the human body and especially to the nervous system is still not conclusive. [1], [2], [3], [4], [5], [6], [7],[8].

The aim of our study was to assess the effects of long term exposure to low power GSM signals on the behaviour and brain cell ultrastructure of rats. The experimental protocol we propose provides for the irradiation of the rats in their daily habitat using the cage top cover as the irradiation antenna.

In order to assess the effects of MW exposure, an open field test (OFT) and an elevated plus maze test (EPM) were performed in order to observe potential behavioral changes. In our study, samples of the hippocampus and the frontal cortex were analyzed by transmission electron microscopy (TEM) in order to find possible ultra-structural changes in these brain regions.

\section{MATERIALS AND METHODS}

A total of 30 (20 exposed to $\mathrm{MW}$ radiation, 10 controls) male Albino Wistar rats were used in this experiment. At the beginning of the experiment, rats were 3 months old, weighing $230 \pm 25 \mathrm{~g}$. The animals were kept in standard plastic cages, 5 animals per cage at a room temperature of $23 \pm 0.5^{\circ} \mathrm{C}$ with a $12 / 12$ hours light/dark cycle, under similar conditions of acoustic noise and ventilation. Irradiated animals and the control group animals were hosted in separate rooms. All animals received standard commercial rodent diet and water ad libitum during the experiment.

Each animal received an identification number at the beginning of the experiment. Numbers were randomly assigned to the MW exposed groups (two group of $n=10$ ) and to the control groups (two groups of $n=5)$. After 3 and 9 months, respectively, 10 animals

\footnotetext{
"danadabala@gmail.com
} 
from the MW exposed group and 5 animals from the control group were tested in terms of behavior and TEM. The animals were tested using blind experiments for locomotor activity one day after the last exposure and for anxiety-like behavior and locomotor activity two days after the last exposure.

On the third day after the last exposure, the animals were anesthetized with a ketamine-xylazine cocktail $(60 \mathrm{mg}$ ketamine and $7.5 \mathrm{mg}$ xylazine respectively/ $\mathrm{kg}$ b.w.). Under anesthesia, animals were euthanized by decapitation in order to collect cerebral tissue for TEM examination.

\subsection{GSM exposure}

The animals were exposed to MW radiation in the $860-890 \mathrm{MHz}$ frequency range, corresponding to the Global System for Mobile Communications GSM-900 uplink (i.e. mobile phone to base) band. The exposure time was 4 hours per day, from 04:00 to 08:00, for 36 weeks. The MW field power density and whole body SAR (Specific Absorption Rate) values varied between $5-120 \mathrm{~mW} / \mathrm{m}_{2}$ and $0.0008-0.3 \mathrm{~W} / \mathrm{kg}$, respectively. The medium exposure value of microwave field power density was $\approx 60 \mathrm{~mW} / \mathrm{m} 2$ and the medium whole body $\mathrm{SAR} \approx 0.15 \mathrm{~W} / \mathrm{kg}$.

\subsection{Experimental setup}

The experiment was performed taking into consideration the International Commission on NonIonizing Radiation Protection (ICNIRP) recommendations regarding the occupational (SAR < $0.4 \mathrm{~W} / \mathrm{kg}$ ) and the general public (SAR $<0.08 \mathrm{~W} / \mathrm{kg})$ exposure limits to EMF in the $10 \mathrm{MHz}-10 \mathrm{GHz}$ frequency range [9],[10],[11]. A typical rat cage, i.e. a transparent plastic box with a metallic grating cover (Figure 1), was adapted to serve as the MW exposure unit. The metallic cover was transformed into an omnidirectional antenna for MW radiation. An Agilent N1911A powermeter with an E9321A peak and average power sensor was used for the initial power characterization and the periodical checking of the MW power generated by the antenna (Figure 2). The rats were situated in the near field of the transmitting antenna. The MW generator was turned on for 4 hours per day, during the rats' active cycle, by a timer switch. Four identical MW exposure units as described above were used in the experiment, with five rats in each unit (Figure 2).

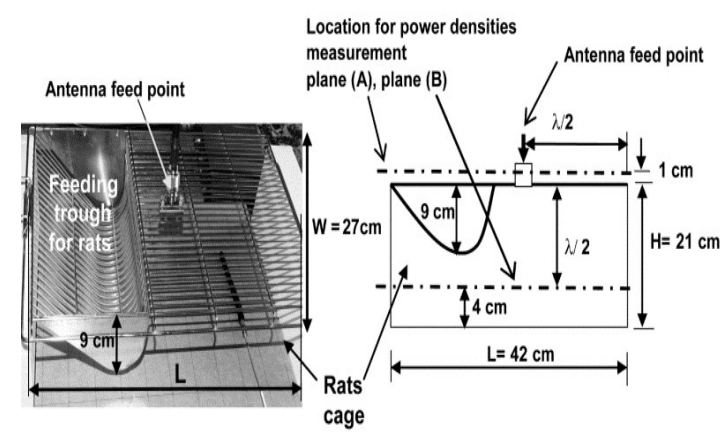

Figure 1. Antenna configuration and locations for MW power density measurements in the rats' cage

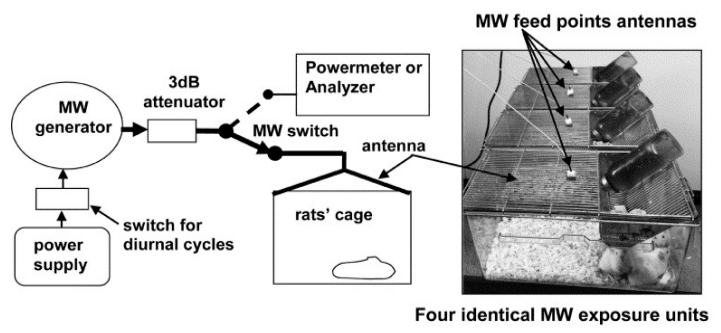

Figure 2.The experimental setup for the MW irradiation of rats

\subsection{Microwave field power density} characterization

The MW power density (PD) distribution measured in the cage is presented in Figure 3.

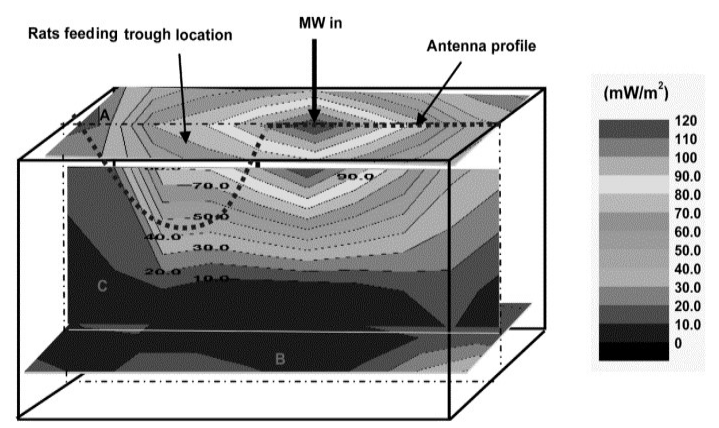

Figure 3. MW field power density distributions in the cage:

(A) close to the antenna ( $1 \mathrm{~cm}$ above the antenna);(B) at $4 \mathrm{~cm}$ from the bottom; (C) in the vertical central plane

\subsection{Behavioral Testing}

\subsubsection{OFT and EPM methods}

Two different tests, largely accepted for studying animal behavior, were used in this study in order to assess the locomotor activity and anxiety-like behavior in rodents: the Open Field Test (OFT) and the Elevated Plus Maze (EPM). Testing was conducted each time between 09:00 and 14:00.

A visual tracking system (Smart Basic Software version 3.0 Panlab Harvard Apparatus) automatically recorded the animal behavior over a 5 minute period, while the rats freely explored the specific mazes (Ugo Basil Animal Mazes for Video-Tracking). After the test, the system automatically generated a folder containing the results [12],[13],[14].

\subsection{Statistical Analysis}

All statistical analyses were conducted using the ANOVA GraphPad Prism software, version 6.0 (GraphPad, San Diego, California, USA). The results were expressed as the mean \pm standard deviation (SEM). Two way analysis of variance (repeated measures of ANOVA) was used, followed by the Bonferonni's post hoc test in order to determine the statistical significant among two groups. A p value below 0.05 was considered to be statistically significant. 
2.6. Tissue sampling and Transmission electron microscopy (TEM)

After the euthanization of the animals, 2 small samples $1.5 \mathrm{~mm}^{3}$ each were collected from each rat's frontal cortex and hippocampus and were further processed for TEM [15],[16], [17]. Samples were fixed with a $2.7 \%$ glutharaldehyde (Electron Microscopy Sciences, Hatfield, USA) in a $0.1 \mathrm{M}$ phosphate buffer $\mathrm{pH} 7.4$ for 2 hours then washed four times with the same buffer and then postfixed 2 hours with a $1.5 \% \mathrm{OsO}_{4}$ (Sigma-Aldrich, St. Louis, USA) in a $0.15 \mathrm{M}$ phosphate buffer and then finally washed twice with the second buffer for 2 hours. After being washed twice with the second buffer, the samples were dehydrated with an acetone (Nordic Invest SRL, Bucharest, Romania) series, and infiltrated with Epon 812 (Fluka AG, Buchs, Switzerland). One sample of the frontal cortex and one of the hippocampus were analysed for each animal with the other samples preserved as backup. Ultrathin sections were cut from at least two different regions of each sample with glass knives using a LKB Ultrotome III Bromma 8800 ultramicrotome (LKB Produckter AB, Stockholm-Bromma, Sweden) and collected on 300 mesh $\mathrm{Cu}$ grids. The sections were contrasted with a saturated solution of uranyl acetate (Merck, Darmstadt, Germany) in ethanol 50\% (18 min), and a $2.8 \%$ lead citrate (Fluka AG, Buchs, Switzerland) (5 min). Examination was performed with a JEOL JEM 1010 microscope (Jeol Ltd., Tokyo, Japan) operating at $80 \mathrm{kV}$ and the images were photographed with a Mega VIEW III system (Olympus, Soft Imaging System, Münster, Germany). All TEM analyses were blind with regard to test situation.

\section{RESULTS}

\subsection{Behavioral tests}

The effects of MW exposure on the rats' general locomotor activity, tested in both OFT and EPM, are illustrated by Figures 4 and 5 .

In OFT, our results showed that 3 months exposure to MW significantly decreased the locomotor activity of rats (total travelled distance and travelled distance in periphery, $\mathrm{p}<0.01$; total number of entries, $\mathrm{p}=0.001$; number of entries in periphery, $\mathrm{p}<0.001$ ).

After 9 months of exposure, the animals travelled significantly less and made fewer entries in OFT (total travelled distance and total number of entries, $\mathrm{p}<$ 0.05 ; travelled distance in periphery, $\mathrm{p}=0.05$; number of entries in periphery, $\mathrm{p}=0.01$ ).

In EPM, neither 3 nor 9 months of exposure to MW statistically influenced the total travelled distance and the travelled distance in the closed arms ( $p>0.05$ ), even though both parameters tended to decrease in the exposed groups. On the other hand, the exposure of the rodents to MW significantly diminished the total number of entries (3 and 9 months) as well as the entries into the closed arms ( 9 months) $(\mathrm{p}<0.05)$.
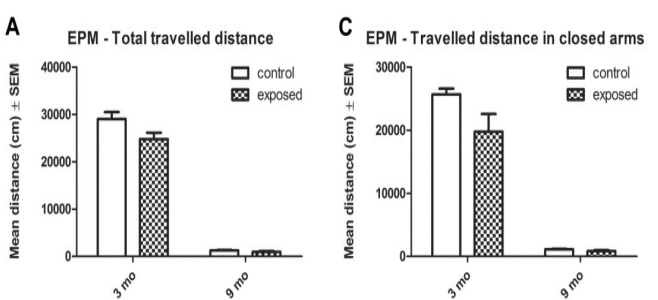

B

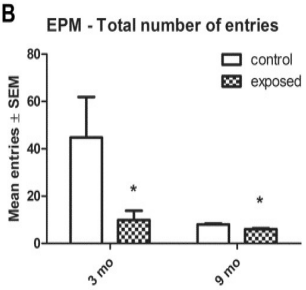

D

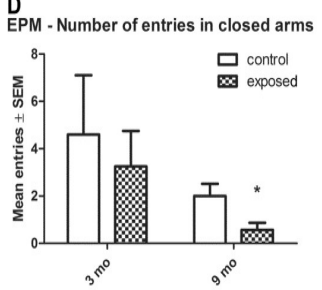

Figure 4. The effects of GSM radiation on total (A) and peripheral (B) travelled distance and total (C) and peripheral (D) number of entries in open field test (OFT).Each exposed group consisted of 10 rats and each control group consisted of 5 rats. Results are expressed as mean \pm SEM.
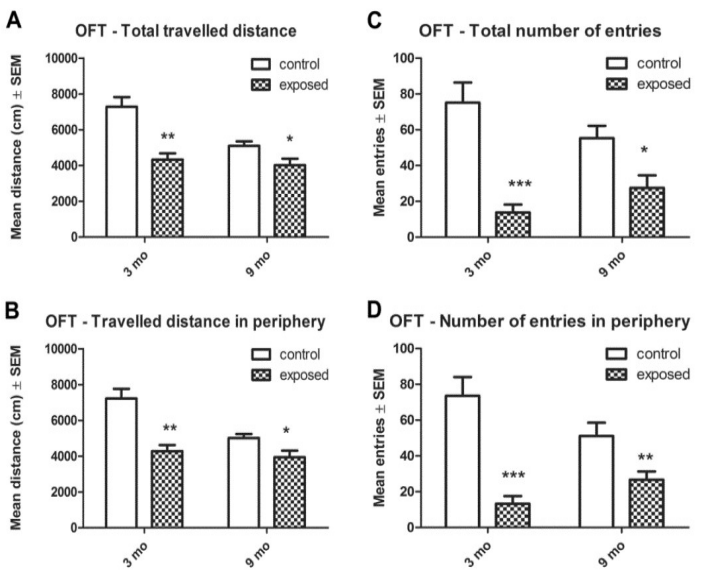

Figure 5. The effects of GSM radiation on the total (A) and peripheral (C) travelled distance and the total (B) and peripheral (D) number of entries in elevated plus maze (EPM). Each group consisted of 10 rats and each control group consisted of 5 rats. Results are expressed as mean \pm SEM; ${ }^{*} \mathrm{p}<0.05,{ }^{* *} \mathrm{p}<0.01,{ }^{* * *} \mathrm{p}<0.001$ as compared with control.

The effect of MW on rats' emotionality, tested in OFT and EPM, is illustrated by Figure 6. In OFT, both the activity (travelled distance and number of entries in center) and the time ratio in the centre of the open field arena tended to diminish in the case of the exposed rats (3 and 9 months) as compared with the control group, but the differences were not statistically significant ( $\mathrm{p}>0.05)$.

In EPM, the groups that were exposed to MW for either 3 or 9 months made significantly fewer entries into the open arms of the elevated maze $(\mathrm{p}<0.05)$. 


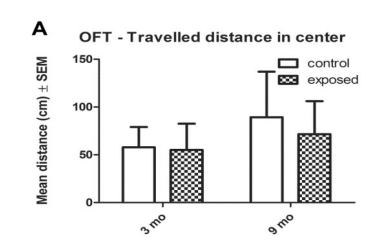

B OFT - Number of entries in center

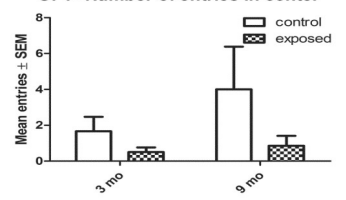

C
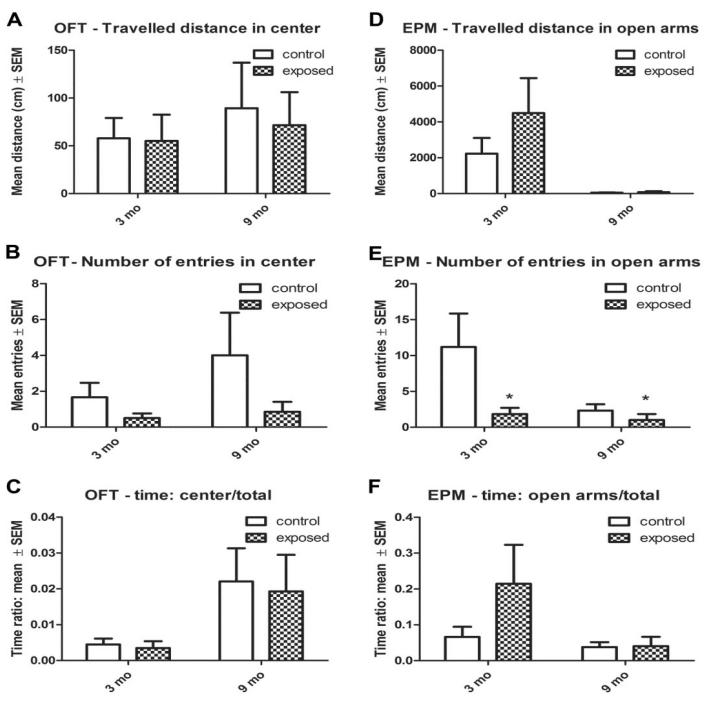

Figure 6. The effects of GSM radiation on emotionality in open field test (OFT) (A, B, C) and in elevated plus maze (EPM) (D, E, F). Each group consisted of 10 rats and each control group consisted of 5 rats. Results are expressed as mean \pm SEM; ${ }^{*} \mathrm{p}<0.05,{ }^{* *} \mathrm{p}<0.01,{ }^{* * *} \mathrm{p}<0.001$ as compared with control.

\subsection{Tissue sampling and Transmission electron microscopy (TEM)}

When sections from the different groups were examined, general aspects and the detailed ultrastructure of neurons, oligodendrocytes, and blood capillaries were taken into consideration. The most common ultrastructural aspects observed in each group are illustrated by Figures 7-12.

\subsubsection{Frontal cortex: control groups (Fig.7)}

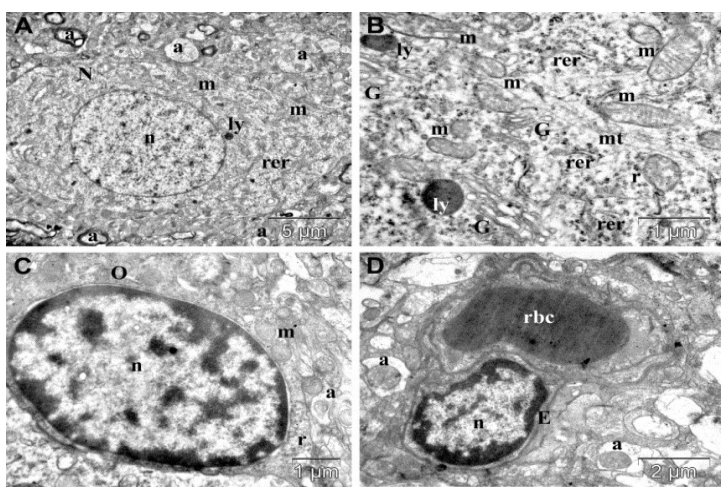

Figure 7. TEM images showing the typical architecture of the frontal cortex in control group $(n=5)$. General A) and detailed (B) views of a neuron, oligodendrocyte (C) and blood capillary

(D). N, neuron; n, nuclei; a, axons; $\mathrm{m}$, mitochondria; rer, rough endoplasmic reticulum; ly, lysosomes; $\mathrm{mt}$

microtubules; G, Golgi apparatus; O, oligodendrocyte; r, ribosomes; E, endothelial cell; rbc, red blood cell

\subsubsection{Frontal cortex: 3 months exposure}

After 3 months of daily exposure to the MW field important ultrastructural degenerative changes were identified in the frontal cortex samples of all tested animals. The neurons showed various degrees of 198 alterations. The most affected ones had highly indented nuclei but still contained prominent nucleoli (Fig. $8,8 \mathrm{~A})$.

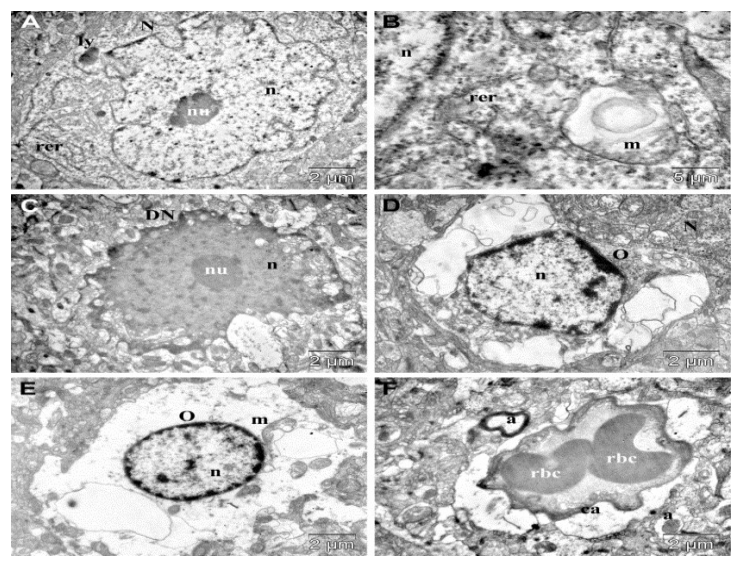

Figure 8. TEM images of the frontal cortex in animals exposed for 3 months to GSM radiation $(n=10)$. Degenerated neuron with indented nucleus (A). Swollen mitochondrion with electron lucent matrix and abnormal, circular cristae (B).

Dark neuron with irregular nucleus cytoplasmatic

vacuolations (C). Highly altered (D) and necrosed (E) oligodendrocytes surrounded by extracellular vacuolations.

Capillary with normal endothelium surrounded by perivascular oedema due mainly to enlargement of the end feet of astrocyte (F). N, neurons; n, nuclei; nu, nucleoli; a, axons; $\mathrm{m}$, mitochondria; rer, rough endoplasmic reticulum;

ly, lysosomes; DN, dark neuron; O, oligodendrocytes; ca, capillary; rbc, red blood cells.

\subsubsection{Frontal cortex: 9 months exposure}

The effects of 9 months exposure to the MW radiation were even more severe in all animals of this group (Fig.9).

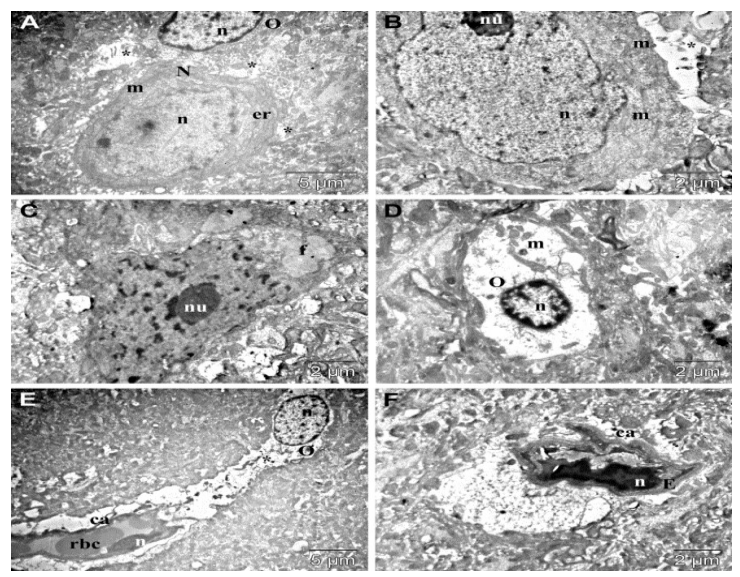

Figure 9. TEM images of the frontal cortex in animals exposed for 9 months to GSM radiation( $\mathrm{n}=10)$. Rarefaction areas $(*)$ of the frontal cortex tissue and altered neurons and oligodendrocytes (A,B). Dark neuron with indented nucleus and increased heterochromatin, and with cytoplasmic deposits of fibrillar inclusions (C). Necrosed oligodendrocytes

(D,E), continued with perivascular oedema (E). Blood capillary with normal aspect, but surrounded by large electron transparent areas (E,F). N, neurons; n, nuclei; nu, nucleoli; $\mathrm{m}$, mitochondria; er, endoplasmic reticulum; $\mathrm{O}$, oligodendrocytes; ca, capillaries; E, endothelial cell; rbc, red blood cells. 


\subsubsection{Hippocampus: Control group (Fig.10)}

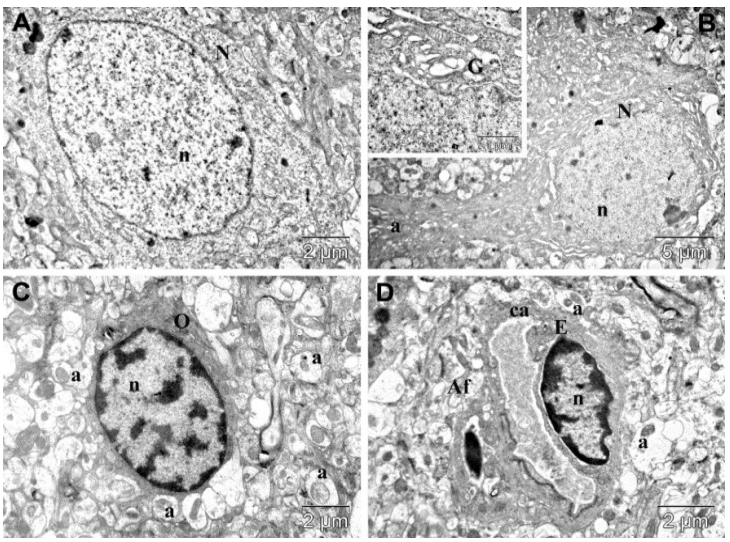

Figure 10. TEM images showing the typical architecture of hippocampus in control group $(n=5)$. General views of neurons (A,B), oligodendrocyte (C) and blood capillary (D) $\mathrm{N}$, neurons; $\mathrm{n}$, nuclei; a, axons; $\mathrm{m}$, mitochondria; $\mathrm{G}$, Golgi apparatus; O, oligodendrocyte; ca, capillary; E, endothelial cell; Af, astrocyte feet.

\subsubsection{Hippocampus: 3 months exposure}

The density of the nervous tissue was extensively reduced in the hippocampus sections as a consequence of astrocytes necrosis and extracellular vacuolation, in all rats of this group (Fig. 11).

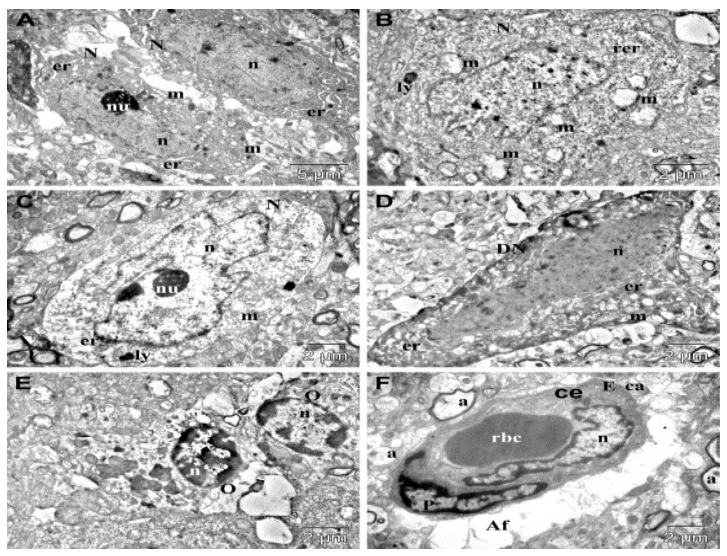

Figure 11. TEM images of hippocampus in animals exposed for 3 months to GSM radiation(n=10). Neurons with major shape alterations containing swollen mitochondria with highly altered cristae (A-C). Dark neurons with cytoplasm vacuolation (D). Normal and necrosed oligodendrocytes (E). Capillary with normal aspect, surrounded by dilated end feet of astrocytes (F). N, neurons; n, nuclei; nu, nucleoli; a, axons; $\mathrm{m}$, mitochondria; er, endoplasmic reticulum; rer, rough endoplasmic reticulum; ly, lysosome; DN, dark neuron; O, oligodendrocytes; ca, capillary; E, endothelial cell; ce, centrioles; rbc, red blood cells; Af, astrocyte feet.

\subsubsection{Hippocampus: 9 months exposure}

Both neurons and olygodendrocytes suffered ultrastructural degenerations in this group (Fig. 12).

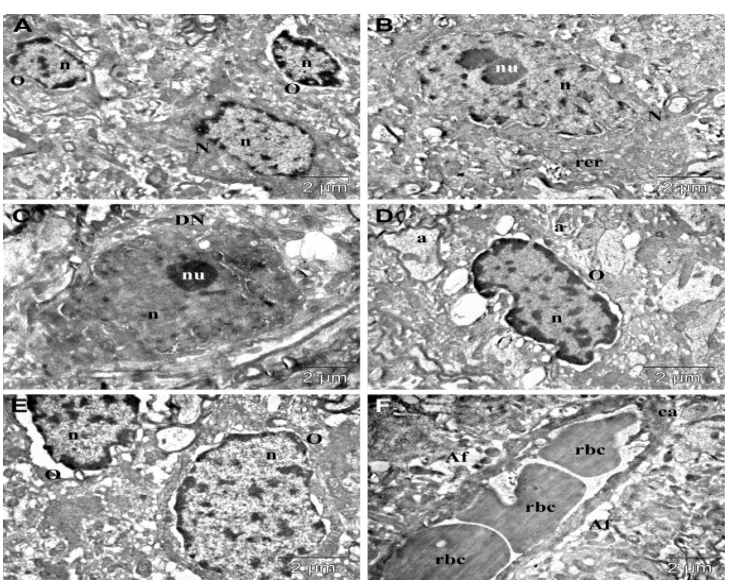

Figure 12. TEM images of hippocampus in animals exposed for 9 months to GSM radiation( $\mathrm{n}=10$ ). Highly degenerated neurons $(\mathrm{A}, \mathrm{B})$ and olygodendrocytes $(\mathrm{A}, \mathrm{D}, \mathrm{E})$. Dark neuron with indented nucleus and with a few organelles (C). Normal blood capillary surrounded by dilated end feet of astrocytes

(F). N, neurons; n, nuclei; nu, nucleoli; a, axons; m, rer, rough endoplasmic reticulum; DN, dark neuron; $\mathrm{O}$,

oligodendrocytes; ca, capillary; rbc, red blood cells; Af, astrocyte feet.

\section{CONCLUSIONS}

Despite being examined by numerous experimental studies, the cognitive function in relationship with GSM MW exposure is highly controversial. Although there is increasing evidence regarding biological effects caused by weak radiofrequency radiations (microwaves) there is a lack of indisputable available data and this matter has not been settled so far [18], [19], [20] ,[21], [22], [23], [24].

Our study highlights that long term exposure to MW radiation in the $860-890 \mathrm{MHz}$ frequency range, corresponding to the GSM-900 uplink band, may have neurobiological effects. The results of the present study suggest that long-term and low-level cumulative MW radiation could cause significant ultrastructural damage at the cerebral substructural level which could trigger behavioral alterations. Our findings indicate that cell injuries could be directly related to the exposure time, increasing in severity as the duration of exposure increases. This issue should warrant special consideration as neuronal damage may not have immediate demonstrable consequences, but might in time lead to late degenerative nervous diseases. We aim to replicate our findings in a more advanced future study in order to elucidate the fundamental mechanisms underlying the interaction between MW radiation and the central nervous system at the cellular and molecular level. Based on our results, further behavioral studies are also needed in order to investigate the effect of MW on the animals' cognitive performance.

Acknowledgements: For the INCDTIM-affiliated authors, this work was supported by the Romanian Ministry of Research and Innovation (MCI) under the Core Programme no. 19N/2016, code PN16-3O. 
REFERENCES

1. H. Lai, A. Horita, A. W. Guy, "Microwave irradiation affects radial-arm maze performance in the rat," Bioelectromagnetics, vol. 15, no. 2, pp. 95 - 104, 1994.

DOI: $10.1002 /$ bem.2250150202

PMid: 8024608

2. B. Wang, H. Lai, "Acute exposure to pulsed 2450-MHz microwaves affects water-maze performance of rats," Bioelectromagnetics, vol. 21, no. 1, pp. $52-56$, Jan. 2000.

DOI: $10.1002 /($ SICI)1521-

186X(200001)21:1<52::AID-BEM8>3.0.CO;2-6 PMid: 10615092

3. B. L. Cobb, J. R. Jauchem, E. R. Adair, "Radial arm maze performance of rats following repeated low level microwave radiation exposure," Bioelectromagnetics, vol. 25, no. 1, pp. $49-57$, Jan. 2004.

DOI: $10.1002 /$ bem.10148

PMid: 14696053

4. Z. J. Sienkiewicz, R. P. Blackwell, R. G. Haylock, R. D. Saunders, B. L. Cobb, "Low-level exposure to pulsed $900 \mathrm{MHz}$ microwave radiation does not cause deficits in the performance of a spatial learning task in mice," Bioelectromagnetics, vol. 21, no. 3, pp. $151-158$, Apr. 2000.

DOI: $10.1002 /($ sici) $1521-$

186x(200004)21:3<151::aid-bem1>3.0.co;2-q PMid: 10723014

5. D. Dubreuil, T. Jay, J. M. Edeline, "Does head-only exposure to GSM-90o electromagnetic fields affect the performance of rats in spatial learning tasks?," Behav. Brain Res., vol. 129, no. 1-2, pp. $203^{-}$ 210, Feb. 2002.

DOI: 10.1016/s0166-4328(o1)oo344-8

PMid: 11809512

6. H. Yamaguchi et al., "1439 $\mathrm{MHz}$ pulsed TDMA fields affect performance of rats in a T-maze task only when body temperature is elevated," Bioelectromagnetics, vol. 24, no. 4, pp. $223-230$, May 2003.

DOI: $10.1002 /$ bem.10099

PMid: 12696082

7. M. P. Ntzouni, A. Stamatakis, F. Stylianopoulou, L. H. Margaritis, "Short-term memory in mice is affected by mobile phone radiation," Pathophysiology, vol. 18, no. 3, pp. 193 - 199, Jun. 2011.

DOI: 10.1016/j.pathophys.2010.11.001 PMid: 21112192

8. J. C. Cassel, B. Cosquer, R. Galani, N. Kuster, "Whole-body exposure to 2.45 GHz electromagnetic fields does not alter radial-maze performance in rats," Behav. Brain Res., vol. 155, no. 1, pp. $37-43$, Nov. 2004.

DOI: 10.1016/j.bbr.2004.03.031

PMid: 15325777

9. ICNIRP guidelines for limiting exposure to timevarying electric, magnetic, and electromagnetic fields (up to $300 \mathrm{GHz}$ ), ICNIRP, Oberschleissheim, Germany, 1988.

Retrieved from:

https://www.icnirp.org/cms/upload/publications/I CNIRPemfgdl.pdf Retrieved on: Jan 27, 2019

10. ICNIRP Statement on the "Guidelines for limiting exposure to time-varying electric, magnetic, and electromagnetic fields (up to $300 \mathrm{GHz}$ )", ICNIRP, Oberschleissheim, Germany, 2009. Retrieved from:

https://www.icnirp.org/cms/upload/publications/ICNI RPStatementEMF.pdf

Retrieved on: Aug. 1, 2019
11. Exposure to high frequency electromagnetic fields, biological effects and health consequences (100 $k H z-300 \mathrm{GHz}$, ICNIRP 16/2009, ICNIRP, Oberschleissheim, Germany, 2009.

Retrieved from: http://ocpm.qc.ca/sites/ocpm.qc.ca/files/pdf/P52/ 5z.pdf

Retrieved on: May 8, 2019

12. M. T. Gamberini, D. S. Rodrigues, D. Rodrigues, V.B. Pontes, "Effects of the aqueous extract of Pimpinella anisum L. seeds on exploratory activity and emotional behavior in rats using the open field and elevated plus maze tests," J. Ethnopharmacol., vol. 168, pp. 45 - 49, Jun. 2015.

DOI: 10.1016/j.jep.2015.03.053 PMid: 25839118

13. A. A. Walf, C. A. Frye, "Antianxiety and antidepressive behavior produced by physiological estradiol regimen may be modulated by hypothalamic-pituitary-adrenal axis activity," Neuropsychopharmacology, vol. 30, no. 7, pp. 1288 - 1301, Jul. 2005.

DOI: $10.1038 /$ sj.npp.1300708 PMid: 15756306

14. A. A. Walf, C. A. Frye, "The use of the elevated plus maze as an assay of anxiety-related behavior in rodents," Nat. Protoc., vol. 2, no. 2, pp. $322-328$, Mar. 2007.

DOI: $10.1038 /$ nprot.2007.44

PMid: 17406592

PMCid: PMC3623971

15. M. A Hayat, Principles and Techniques of Electron Microscopy: Biological Applications, 4th ed., Cambridge, UK: Cambridge Univ. Press, 2000. Retrieved from:

https://archive.org/stream/PrinciplesTechniquesO fElectronMicroscopyVolume2/HayatPrinciplesTechniquesOfElectronMicroscopyVol2 d jvu.txt

Retrieved on: Mar. 29, 2019

16. W. Bloom, D. W. Fawcet, "The nervous tissue" in A textbook of Histology, 10th ed., Philadelphia (PA), USA: W. B. Saunders Co., 1975, ch. 12, pp. $333-363$.

Retrieved from:

https://trove.nla.gov.au/work/11587217?q\&sort=h $\underline{\text { oldings }+ \text { desc } \&=1574538277129 \& \text { versionId }=45416}$ $\underline{250}$

Retrieved on: Dec. 29, 2018

17. I. M. Watt, The Principles and Practice of Electron Microscop, 2nd ed., Cambridge, UK: Cambridge Univ. Press, 1997.

Retrieved from: https://www.scribd.com/document/337211592/Th e-Principles-and-Practice-of-Electron-Microscopy Retrieved on: Sep. 13, 2019

18. A. İkinci et al., "The Effects of Prenatal Exposure to a 900 Megahertz Electromagnetic Field on Hippocampus Morphology and Learning Behavior in Rat Pups," NeuroQuantology, vol. 11, no. 4, pp. $582-590$, Dec. 2003.

DOI: $10.14704 / \mathrm{nq} .2013 .11 .4 .699$

19. H. S. Aboul Ezz, Y. A. Khadrawy, N. A. Ahmed, N. M. Radwan, N. M. El Bakry, "The effect of pulsed electromagnetic radiation from mobile phone on the levels of monoamine neurotransmitters in four different areas of rat brain," Eur. Rev. Med. Pharmacol. Sci., vol. 17, no. 13, pp. $1782-1788$, Jul. 2013.

PMid: 23852905

20. K. Maaroufi et al., "Spatial learning, monoamines and oxidative stress in rats exposed to $900 \mathrm{MHz}$ electromagnetic field in combination with iron overload," Behav. Brain Res., vol. 258, pp. 80 - 89, Jan. 2014. 
DOI: 10.1016/j.bbr.2013.10.016

PMid: 24144546

21. I. Pavacic, I. Trosic, "In vitro testing of cellular response to ultra high frequency electromagnetic field radiation," Toxicol. In Vitro., vol. 22, no. 5, pp. 1344 - 1348, Aug. 2008.

DOI: 10.1016/j.tiv.2008.04.014

PMid: 18513921

22. V. S. S. S. Sajja, N. Hlavac, P. J. VandeVord, "Role of Glia in Memory Deficits Following Traumatic Brain Injury: Biomarkers of Glia Dysfunction," Front. Integr. Neurosci., vol. 10, article no. 7, Feb. 2016.

DOI: 10.3389/fnint.2016.00007

PMid: 26973475

PMCid: PMC4770450
23. L. G. Salford, A. E. Brun, J. L. Eberhardt, L. Malmgren, B. R. Persson, "Nerve cell damage in mammalian brain after exposure to microwaves from GSM mobile phones," Environ. Health Perspect., vol. 111, no. 7, pp. $881-883$, Jun. 2003. DOI: 10.1289/ehp.6039

PMid: 12782486 PMCid: PMC1241519

24. J. Tang et al., "Exposure to $900 \mathrm{MHz}$ electromagnetic fields activates the mkp-1/ERK pathway and causes blood-brain barrier damage and cognitive impairment in rats," Brain Res., vol. 1601, pp. 92 - 101, Mar. 2015.

DOI: 10.1016/j.brainres.2015.01.019

PMid: 25598203 